\title{
Simulating Multi-tone Free-running Oscillators with Optimal Sweep Following
}

\author{
S.H.M.J. Houben*
}

July 1, 2002

\begin{abstract}
A new method for the simulation of circuits with widely-varying time scales is given. The method makes a splitting of the behaviour of the circuit in a fastvarying and a slowly-varying component. The method is attractive because it can handle frequency modulated (FM) circuits, unlike existing methods. Numerical results are given.
\end{abstract}

\section{Introduction}

With ever increasing operating frequencies of electric circuits, widely varying time scales become increasingly common. The fast frequency $f_{f}$ can be up to $10^{3}$ times the slow frequency $f_{s}$. A typical example would be a circuit which generates a highfrequency carrier wave, which is modulated with a low-frequency data signal. In such a situation, the period and/or amplitude changes of the high-frequency signal changes slowly compared to the high-frequency oscillation itself.Traditional simulation techniques have great difficulty with such widely separate time scales, since they require an amount of computation time $O\left(f_{f} / f_{s}\right)$. In order to simulate a complete waveform of the low-frequency signal, such techniques have to simulate many waveforms of the high-frequency signals.

Several approaches have been suggested to speed up the simulation of such a circuit with widely varying time scales. They can be split into two groups.

1. Methods that are based on partitioning a circuit into a fast and a slow part, such as those described in [1]. These methods can be very effective when a good partitioning can be made, but this is not always possible.

2. Methods that simulate the circuit as a whole, but attempt to split the low-frequency behaviour from the high-frequency behaviour. Examples of such methods are envelope-following techniques [3, 4], and the Multi-rate Partial Differential Equation (MPDE) technique $[2,6]$.

In this paper, a new method of type 2 is discussed. The method is named Optimal Sweep Following New in this method is that it handles autonomous circuits in which Frequency Modulation (FM) takes place. MPDE and Envelope Following only handle Amplitude Modulated (AM) circuits.

\footnotetext{
*Eindhoven University of Technology, e-mail: stephanh@win.tue.nl
} 


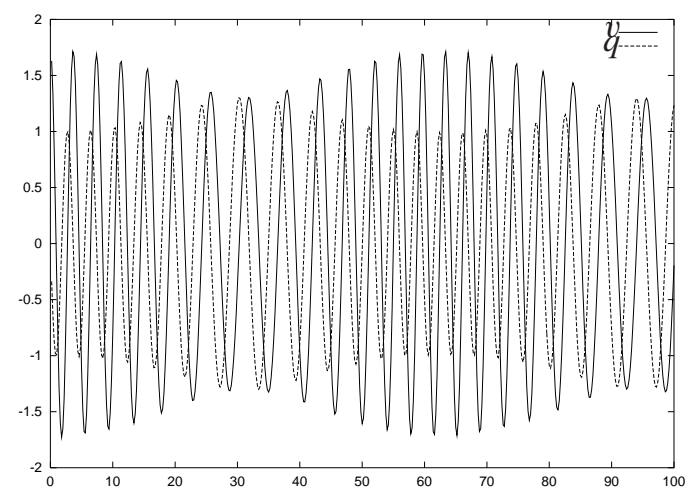

Figure 1: Transient solution of a modulated LC-oscillator

The Sweep Following method splits the behaviour of the system in a slowly-varying and a fast-varying component. The splitting is optimal in its class.

The essential new feature of the method is that the frequency of the fast-varying signal does not have to be provided a priori; rather, it is determined by the method itself. As a result, the method can detect and handle changes in the period of the fastvarying signal. This makes it suitable for simulating Frequency Modulated circuits. Other methods require the period $f_{f}$ to be provided a priori, and cannot deal with the situation where $f_{f}$ changes over time. The fact that our method computes $f_{f}$ locally is also an advantage in that this information is often desired by the circuit designer.

This paper is built up as follows. In section 2, we explain the notion of a sweep and show how a sweep can efficiently be represented. In section 3, we outline the Sweep Following method. Finally, in section 4 we present a test problem and show that Sweep Following solves it. The test problem is an FM-modulated problem; it cannot be handled by MPDE or Envelope Following.

\section{Efficient representation of solutions with widely-varying time-scales}

Consider the following formulation of a Differential-Algebraic Equation (DAE).

$$
\frac{d}{d t} \mathbf{q}(\mathbf{x})+\mathbf{j}(\mathbf{x})=\mathbf{s}(t) .
$$

The following definition is often importance throughout this paper.

Definition 1. A sweep of a set $\mathcal{C}_{0} \subset \mathbf{R}^{N}$ by (1) is the set of points $\left\{\xi\left(\mathbf{x}_{0}, t\right) \mid \mathbf{x}_{0} \in\right.$ $\left.\mathcal{C}_{0}, t \geq 0\right\}$, where $\xi\left(\mathbf{x}_{0}, t\right)$ is defined as the solution of (1) with initial condition $\mathbf{x}(0)=$ $\mathbf{x}_{0}$ at time $t$.

The solution $\mathbf{x}$ of (1) may change at two very different time scales, as is the case in Fig. 1. Thus we have a fast oscillation, which itself slowly changes due to the lowfrequency signal. This gives rise to "telephone cord" solutions as shown in Fig. 2. We call these "telephone cord" solutions because they are tightly wound in a spiral, just like the cord of a telephone. The key idea of the Sweep Following method is not to simulate the "telephone cord" itself, but rather the following two entities. 


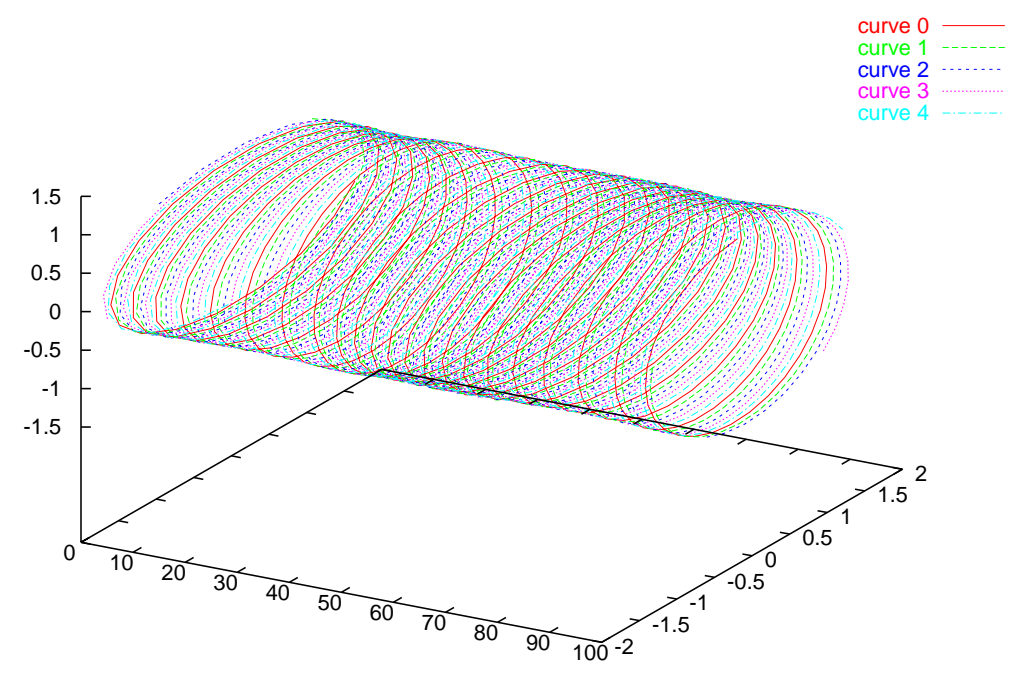

Figure 2: Solutions of the modulated LC-oscillator in $(v, q, t)$-space for various initial conditions.

1. The "tube" that is spanned by the cord.

2. The speed of the rotation of the cord around the tube.

It turns out that it is often cheaper to represent tube and speed rather than to directly represent the solution ("telephone cord") itself.

We now give a rigorous definition of the "tube" that represents the solution 2. Suppose we have a set $\mathcal{C}_{0}$ of initial values to (1), parametrised by a parameter $s \in[0,2 \pi]$, i.e. $\mathcal{C}_{0}=\left\{\hat{\mathbf{x}}_{0}(s) \mid 0 \leq s \leq 2 \pi\right\}$. Moreover, we require that the parametrisation $\hat{\mathbf{x}}_{0}$ is continuous, differentiable and periodic with period $2 \pi$. In addition, the right-hand side $\mathbf{s}$ is also a continuous, differentiable function of $s$ and $t$.

For a given $s \in[0,2 \pi]$, consider the solution $\hat{\mathbf{x}}(s, t)$ to (1) with initial condition $\hat{\mathbf{x}}_{0}(s)$, i.e. the solution of the problem

$$
\begin{aligned}
\frac{d}{d t} \mathbf{q}(\hat{\mathbf{x}}(s, t))+\mathbf{j}(\hat{\mathbf{x}}(s, t)) & =\mathbf{s}(s, t), \\
\hat{\mathbf{x}}(s, 0) & =\hat{\mathbf{x}}_{0}(s) .
\end{aligned}
$$

The function $\hat{\mathbf{x}}$ depends on two variables $s$ and $t$ and parametrises a surface on which all solutions of (1) that have initial values in $\mathcal{C}_{0}$. This surface is the "tube" discussed earlier. Observe that this tube is exactly the sweep of $\mathcal{C}_{0}$ by (1). Since our method follows this sweep, rather than a single solution, it is called "Sweep Following". The parametrisation $\hat{\mathbf{x}}$ of the sweep induces a coordinate system $(s, t)$ on the sweep. However, this coordinate system may be very skew. This implies that information is not efficiently represented in this coordinate system. Therefore, we investigate coordinate transformations of the form

$$
u=s+\alpha(t), \quad v=t,
$$


for some differentiable function $\alpha$. The parametrisation in $(u, v)$ coordinates is called $\hat{\mathbf{x}}_{\alpha}$. With this definition we have

$$
\begin{aligned}
\hat{\mathbf{x}}_{\alpha}(u, v) & =\hat{\mathbf{x}}_{\alpha}(s+\alpha(t), t):=\hat{\mathbf{x}}(s, t), \\
\text { and } \quad \mathbf{s}_{\alpha}(u, v) & =\mathbf{s}_{\alpha}(s+\alpha(t), t):=\mathbf{s}(s, t) .
\end{aligned}
$$

From (4) and (2), we can derive

$$
\begin{aligned}
\frac{d}{d t} \mathbf{q}\left(\hat{\mathbf{x}}_{\alpha}(u, v)\right)+\mathbf{j}\left(\hat{\mathbf{x}}_{\alpha}(u, v)\right) & =\mathbf{s}_{\alpha}(u, v), \\
\hat{\mathbf{x}}_{\alpha}(u, 0) & =\hat{\mathbf{x}}_{0}(s) .
\end{aligned}
$$

By using (3), we can rewrite (6) into

$$
\begin{aligned}
\alpha^{\prime}(v) \frac{\partial}{\partial u} \mathbf{q}\left(\hat{\mathbf{x}}_{\alpha}(u, v)\right)+\frac{\partial}{\partial v} \mathbf{q}\left(\hat{\mathbf{x}}_{\alpha}(u, v)\right)+\mathbf{j}\left(\hat{\mathbf{x}}_{\alpha}(u, v)\right) & =\mathbf{s}_{\alpha}(u, v), \\
\hat{\mathbf{x}}_{\alpha}(u, 0) & =\hat{\mathbf{x}}_{0}(u-\alpha(0)) .
\end{aligned}
$$

The following observations are important to make.

- The resulting formulation is a partial differential equation (PDE) in $u$ and $v$. Obviously, (7) reduces to an ordinary DAE along lines of constant $s$; that is, lines of the form $u-\alpha(v)=$ constant. Lines of this form are the characteristics of (7). See also [5].

- The formulation is not unique: it depends on a certain choice for $\alpha$.

- From the way that (7) has been derived, it follows that it is well posed if and only if (2) is well-posed.

The characteristics are all of the form

$$
u=\alpha(v)+c, \quad \text { for some } c \in \mathbf{R} .
$$

This means that characteristics can never intersect each other, and therefore no shocks in the solution can occur.

\section{Outline of the method}

The basic idea of the method is now as follows. We start at some initial sweep $\mathcal{C}_{0}$. We select a number of points $\hat{\mathbf{x}}_{\alpha}\left(u_{i}, 0\right)$ on $\mathcal{C}_{0}$, and use (7) as an evolution equation in $v$ to find $\hat{\mathbf{x}}_{\alpha}\left(u_{i}, v_{j}\right)$ at later points $\left\{v_{j}\right\}$. Finally, we use the relation (4) to find the solution of the original problem (1).

At first, it may appear that solving (7) is much more expensive than solving (1). However, for a specific class of problems and for a suitable choice of $\alpha$, the solution of (7) changes much slower than the solution of (1). In this case, solving (7) allows much larger step sizes to be taken than if we solve (1). This is the motivating idea behind the method.

This raises of course the question how $\alpha$ should be selected. We want to choose $\alpha$ in such a way that the step-size $\Delta v$ of the time stepping in the $v$ direction can be taken as large as possible, without compromising accuracy. In essence, we want the factor 
$\frac{\partial}{\partial v} \mathbf{q}\left(\hat{\mathbf{x}}_{\alpha}(u, v)\right)$ from (7) to be small; the smaller this factor is, the larger step-sizes $\Delta v$ can be taken. Using (7) we find

$$
\int_{0}^{2 \pi}\left\|\frac{\partial}{\partial v} \mathbf{q}\left(\hat{\mathbf{x}}_{\alpha}(u, v)\right)\right\|^{2} d u=\int_{0}^{2 \pi}\left\|\mathbf{s}_{\alpha}(u, v)-\mathbf{j}\left(\hat{\mathbf{x}}_{\alpha}(u, v)\right)-\alpha^{\prime}(v) \frac{\partial}{\partial u} \mathbf{q}\left(\hat{\mathbf{x}}_{\alpha}(u, v)\right)\right\|^{2} d u .
$$

We want to look for an $\alpha^{\prime}(v)$ for which (9) becomes minimal. Basic calculus shows that this happens when

$$
\alpha^{\prime}(v)=\frac{\int_{0}^{2 \pi}\left(\mathbf{s}_{\alpha}(u, v)-\mathbf{j}\left(\hat{\mathbf{x}}_{\alpha}(u, v)\right), \frac{\partial}{\partial u} \mathbf{q}\left(\hat{\mathbf{x}}_{\alpha}(u, v)\right)\right) d u}{\int_{0}^{2 \pi}\left\|\frac{\partial}{\partial u} \mathbf{q}\left(\hat{\mathbf{x}}_{\alpha}(u, v)\right)\right\|^{2} d u} .
$$

From (7a), we find that

$$
\mathbf{s}_{\alpha}(u, v)-\mathbf{j}\left(\hat{\mathbf{x}}_{\alpha}(u, v)\right)=\alpha^{\prime}(v) \frac{\partial}{\partial u} \mathbf{q}\left(\hat{\mathbf{x}}_{\alpha}(u, v)\right)+\frac{\partial}{\partial v} \mathbf{q}\left(\hat{\mathbf{x}}_{\alpha}(u, v)\right) .
$$

With that, we find that

$$
\begin{aligned}
& \alpha^{\prime}(v)=\frac{\int_{0}^{2 \pi}\left(\alpha^{\prime}(v) \frac{\partial}{\partial u} \mathbf{q}\left(\hat{\mathbf{x}}_{\alpha}(u, v)\right)+\frac{\partial}{\partial v} \mathbf{q}\left(\hat{\mathbf{x}}_{\alpha}(u, v)\right), \frac{\partial}{\partial u} \mathbf{q}\left(\hat{\mathbf{x}}_{\alpha}(u, v)\right)\right) d u}{\int_{0}^{2 \pi}\left\|\frac{\partial}{\partial u} \mathbf{q}\left(\hat{\mathbf{x}}_{\alpha}(u, v)\right)\right\|^{2} d u} \\
&=\alpha^{\prime}(v)+\frac{\int_{0}^{2 \pi}\left(\frac{\partial}{\partial v} \mathbf{q}\left(\hat{\mathbf{x}}_{\alpha}(u, v)\right), \frac{\partial}{\partial u} \mathbf{q}\left(\hat{\mathbf{x}}_{\alpha}(u, v)\right)\right) d u}{\int_{0}^{2 \pi}\left\|\frac{\partial}{\partial u} \mathbf{q}\left(\hat{\mathbf{x}}_{\alpha}(u, v)\right)\right\|^{2} d u}
\end{aligned}
$$

So we see that for this choice of $\alpha^{\prime}$, we obtain

$$
\int_{0}^{2 \pi}\left(\frac{\partial}{\partial v} \mathbf{q}\left(\hat{\mathbf{x}}_{\alpha}(u, v)\right), \frac{\partial}{\partial u} \mathbf{q}\left(\hat{\mathbf{x}}_{\alpha}(u, v)\right)\right) d u=0 .
$$

I.e. there is an orthogonality between $\frac{\partial}{\partial v} \mathbf{q}$ and $\frac{\partial}{\partial u} \mathbf{q}$. Equation (10) gives us a differential equation for $\alpha$. The system formed by (7) and (10) is a complete evolution equation for $\hat{\mathbf{x}}_{\alpha}$ and $\alpha$.

In order to compute a solution of the system (7), (10), we have to discretise the system. We treat $u$ as a space parameter and $v$ as the time parameter. Then we use the method of lines, i.e. we first discretise in the space direction $u$. The resulting semidiscrete system can then be solved in the $v$ direction by a choice of ODE methods.

For the discretisation in the $u$ direction, a number of points $\left\{u_{1}, \ldots, u_{N}\right\}$ is selected. The operator $\frac{\partial}{\partial u}$ can be discretised by a simple symmetric difference scheme. 


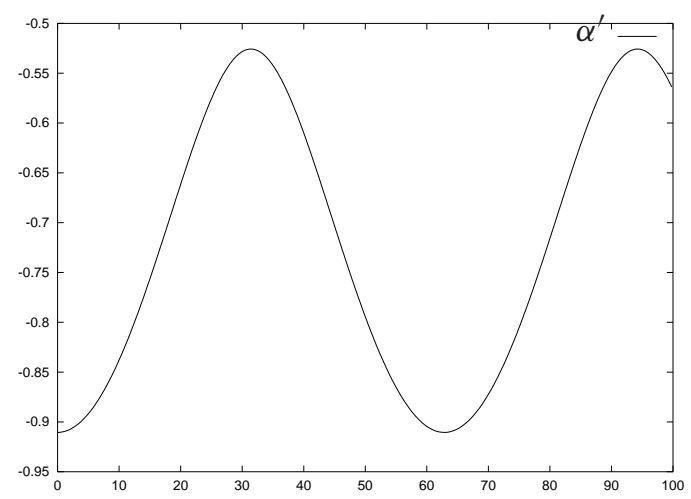

Figure 3: The $\alpha^{\prime}$ computed for (17)

The semi-discretised system now becomes

$$
\begin{aligned}
\frac{\partial}{\partial v} \mathbf{q}\left(\hat{\mathbf{x}}_{\alpha}\left(u_{i}, v\right)\right) & =\mathbf{s}_{\alpha}\left(u_{i}, v\right)-\mathbf{j}\left(\hat{\mathbf{x}}_{\alpha}\left(u_{i}, v\right)\right)-\alpha^{\prime}(v) \mathbf{d}_{i}(v) \text { for } i=1, \ldots, N, \\
\alpha^{\prime}(v) & =\frac{\sum_{i=1}^{N}\left(\mathbf{d}_{i}(v), \mathbf{s}_{\alpha}\left(u_{i}, v\right)-\mathbf{j}\left(\hat{\mathbf{x}}_{\alpha}\left(u_{i}, v\right)\right)\right)}{\sum_{i=1}^{N}\left\|\mathbf{d}_{i}(v)\right\|^{2}} .
\end{aligned}
$$

where the $\mathbf{d}_{i}$ are defined as

$$
\mathbf{d}_{i}(v):=\frac{\mathbf{q}\left(\hat{\mathbf{x}}_{\alpha}\left(u_{i+1}, v\right)\right)-\mathbf{q}\left(\hat{\mathbf{x}}_{\alpha}\left(u_{i-1}, v\right)\right)}{u_{i+1}-u_{i-1}} .
$$

Unfortunately, the computation of $\alpha^{\prime}(v)$ is somewhat expensive, and the computation of $d \alpha^{\prime}(v) / d v$ is even more expensive. Recall that this choice of $\alpha(v)$ was only taken because this leads to a coordinate system that is optimal in some sense. However, if this particular choice is too expensive, it makes sense to take a slightly sub-optimal but much cheaper alternative. This alternative is to keep $\alpha^{\prime}$ constant between two successive time steps $v_{n}$ and $v_{n+1}$, as follows.

$$
\alpha^{\prime}(v)=\frac{\sum_{i=1}^{N}\left(\mathbf{d}_{i}\left(v_{n}\right), \mathbf{s}_{\alpha}\left(u_{i}, v_{n}\right)-\mathbf{j}\left(\hat{\mathbf{x}}_{\alpha}\left(u_{i}, v_{n}\right)\right)\right)}{\sum_{i=1}^{N}\left\|\mathbf{d}_{i}\left(v_{n}\right)\right\|^{2}}, \text { for } v \in\left[v_{n}, v_{n+1}\right) .
$$

\section{Test problem}

A simple test problem has been constructed, which captures the essence of the problems we want to solve. We take a simple modulated LC oscillator. The capacitor is controlled by some external source.

$$
\begin{aligned}
\dot{v} & =C \Theta q, \\
\dot{q} & =-L v, \\
\Theta & =\Theta_{\mathrm{avg}}+\Theta_{\mathrm{amp}} \cos \omega_{\Theta} t .
\end{aligned}
$$

The choice of parameters in the simulations is $L=C=1, \Theta_{\mathrm{avg}}=2, \Theta_{\mathrm{amp}}=1$ and $\omega_{\Theta}=\frac{1}{10}$. A transient simulation of (17) is shown in Fig. 1. We find that if we take $\Theta$ 


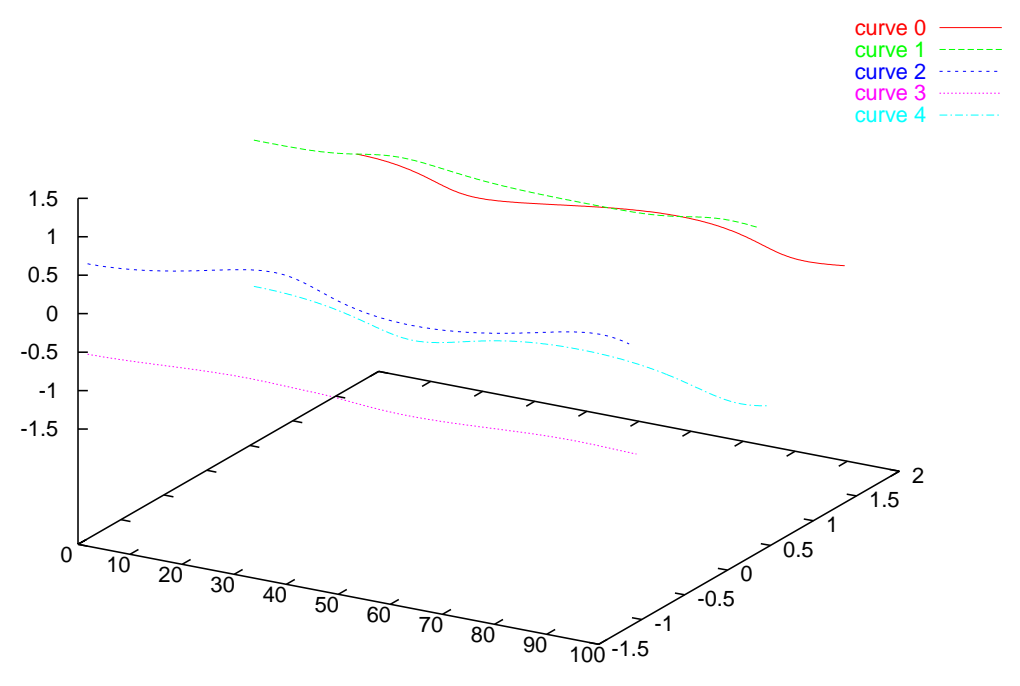

Figure 4: Solution of (17) with sweep following.

to be constant $\Theta_{\text {avg }}+\Theta_{\text {amp }}$ in problem (17), we obtain a solution where $(v, q)$ describe an ellipse around $\mathbf{0}$ with radii 1 and $\sqrt{3}$. This ellipse will be taken as our initial set $\mathcal{C}_{0}$. If we take a few points on this ellipse, and compute the solution for each of these initial values, we obtain Fig. 2. This figure gives a good idea of the sweep that is tracked. Note that the computed solutions all vary very rapidly.

We now apply the Sweep Following idea, i.e. we compute $\alpha^{\prime}$. The computed curve is shown in Fig. 3. This $\alpha^{\prime}$ is then used to compute a solution $\hat{\mathbf{x}}_{\alpha}$, which is shown in Fig. 4. Note the smooth, flat behaviour of $\hat{\mathbf{x}}_{\alpha}$ as a function of $v$. Since the computed curves vary much slower in Fig. 4 than in Fig. 2, we see that it is now possible to take much larger step-sizes in the $v$ direction. This in turn leads to a considerable gain in efficiency.

\section{References}

[1] A. Bartel. Multirate ROW methods of mixed type for circuit simulation. In Scientific Computing in Electrical Engineering. Springer, 2000.

[2] H.G. Brachtendorf, G. Welsch, R. Laur, and A. Bunse-Gerstner. Numerical steady state analysis of electronic circuits driven by multi-tone signals. Electronic Engineering, 79(2):103-112, 1996.

[3] K. Kundert, J. White, and A. Sangiovanni-Vincentelli. An envelope-following method for efficient transient simulation of switching power and filter circuits. In Proc. IEEE Int. Conf. Computer-Aided Design, pages 446-449, 1988.

[4] Kartikeya Mayaram, David C. Lee, Shahriar Moinian, David A. Rich, and Jaijeet Roychowdhury. Computer-aided circuit analysis tools for RFIC simulation: Algo- 
rithms, features and limitations. IEEE Transactions on Circuit and Systems - II: Analog and Digital Signal Processing, 47(4):274-286, 2000.

[5] R. Pulch and M. Günther. A method of characteristics for solving multirate partial differential equations in radio frequency applications. Universität Karlsruhe, Institut für Wissenschaftliches Rechnen und Mathematische Modellbildung, pre-print Nr. 00/07, 2000

[6] Jaijeet Roychowdhury. Analysing circuits with widely-separated time scales using numerical PDE methods. IEEE Transactions in Circuit and Systems - I: Fundamental Theory and Applications, 48(5):578-594, 2001. 